\title{
Culture-dependent and -independent methods reveal dominance of halophilic Euryarcheota in high-altitude Andean Lakes
}

\author{
Marcos J. Maldonado, Virginia H. Albarracín, José A. Lara, Marcela A. Ferrero, \\ María E. Farías*
}

*Corresponding author: mefarias@proimi.org.ar

Aquatic Microbial Ecology 81: 171-188 (2018)

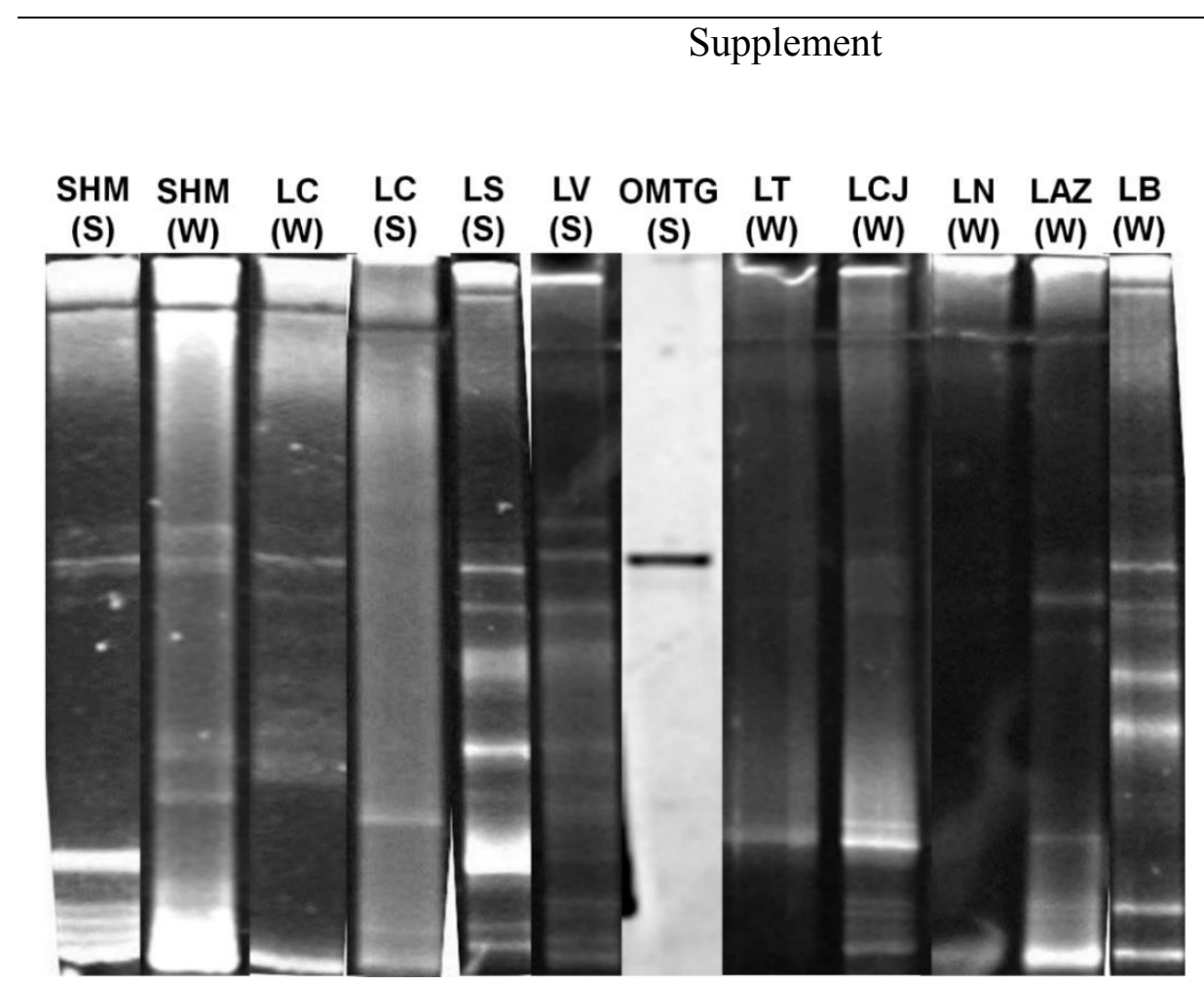

Fig. S1. Denaturing Gradient Gel Electrophoresis from the 12 sampled sites. 
Table S1. Summary characteristics of the studied strains.

\begin{tabular}{|c|c|c|c|c|c|c|c|c|c|c|c|c|c|c|c|c|c|c|}
\hline \multirow[t]{2}{*}{ Strain } & \multicolumn{4}{|c|}{$\% \mathrm{NaCl}$} & \multicolumn{4}{|c|}{ pH } & \multicolumn{2}{|r|}{ UV-B (h) } & \multicolumn{4}{|c|}{ As $[\mathrm{V}](\mathrm{mM})$} & \multicolumn{4}{|c|}{ As [III] $(\mathrm{mM})$} \\
\hline & 5 & 10 & 20 & 30 & 3 & 5 & 8 & 10 & 12 & 24 & 100 & 150 & 200 & 400 & 2.5 & 5 & 7.5 & 10 \\
\hline AC1 & - & ++ & ++++ & ++ & - & ++ & ++++ & ++ & +++ & + & ++++ & +++ & ++ & + & ++++ & ++ & ++ & + \\
\hline AC2 & - & ++ & ++++ & ++ & - & ++ & ++++ & ++ & +++ & + & ++++ & +++ & ++ & + & ++++ & ++ & ++ & + \\
\hline $\mathrm{AC3}$ & - & ++ & ++++ & ++ & - & ++ & ++++ & ++ & +++ & + & ++++ & +++ & ++ & + & ++++ & ++ & ++ & + \\
\hline $\mathrm{AC} 10$ & - & ++ & ++++ & ++ & - & ++ & ++++ & ++ & + & - & +++ & ++ & ++ & + & +++ & ++ & ++ & + \\
\hline AC12 & - & ++ & ++++ & ++ & - & ++ & ++++ & ++ & + & - & +++ & ++ & ++ & + & +++ & ++ & ++ & + \\
\hline AT4 & - & ++ & ++++ & ++ & - & +++ & ++++ & + & + & - & +++ & ++ & ++ & + & +++ & ++ & ++ & - \\
\hline AT5 & - & ++ & ++++ & ++ & - & +++ & ++++ & + & + & - & +++ & ++ & ++ & + & +++ & ++ & ++ & - \\
\hline AT6 & - & ++ & ++++ & ++ & - & +++ & ++++ & + & + & - & +++ & ++ & ++ & + & +++ & ++ & ++ & - \\
\hline AT7 & - & ++ & ++++ & ++ & - & +++ & ++++ & + & + & - & +++ & ++ & ++ & + & +++ & ++ & ++ & - \\
\hline AT8 & - & ++ & ++++ & ++ & - & +++ & ++++ & + & + & - & +++ & ++ & ++ & + & ++++ & ++ & + & - \\
\hline AT9 & - & ++ & ++++ & ++ & - & +++ & ++++ & + & + & - & +++ & ++ & ++ & + & ++++ & ++ & + & - \\
\hline AT14 & - & ++ & ++++ & ++ & - & +++ & ++++ & + & + & - & +++ & ++ & ++ & + & ++++ & ++ & + & - \\
\hline AT 23 & - & ++ & ++++ & ++ & - & +++ & ++++ & + & +++ & + & ++++ & +++ & ++ & + & ++++ & ++ & ++ & + \\
\hline A25 & - & + & ++++ & ++ & - & ++ & ++++ & ++ & +++ & + & ++++ & +++ & ++ & + & ++++ & ++ & ++ & + \\
\hline A26 & - & ++ & ++++ & ++ & - & ++ & ++++ & ++ & +++ & + & ++++ & +++ & ++ & + & ++++ & ++ & ++ & + \\
\hline A29 & - & ++ & ++++ & ++ & - & ++ & ++++ & ++ & +++ & + & ++++ & +++ & ++ & + & ++++ & ++ & ++ & + \\
\hline A30 & - & ++ & ++++ & ++ & - & ++ & ++++ & ++ & +++ & + & ++++ & +++ & ++ & + & ++++ & ++ & ++ & + \\
\hline A31 & - & ++ & ++++ & ++ & - & ++ & ++++ & ++ & +++ & + & +++ & ++ & ++ & + & ++++ & ++ & ++ & + \\
\hline AJ57 & - & ++ & ++++ & ++ & - & ++ & ++++ & ++ & +++ & + & ++++ & +++ & ++ & + & ++++ & ++ & ++ & + \\
\hline AJ58 & - & ++ & ++++ & ++ & - & ++ & ++++ & ++ & +++ & + & ++++ & +++ & ++ & + & ++++ & ++ & ++ & + \\
\hline AJ62 & - & ++ & ++++ & ++ & - & ++ & ++++ & ++ & +++ & + & ++++ & +++ & ++ & + & ++++ & ++ & ++ & + \\
\hline AJ63 & - & ++ & ++++ & ++ & - & ++ & ++++ & ++ & +++ & + & ++++ & +++ & ++ & + & ++++ & ++ & ++ & + \\
\hline AJ67 & - & + & ++++ & ++ & - & ++ & ++++ & ++ & +++ & ++ & ++++ & +++ & ++ & + & ++++ & ++ & ++ & + \\
\hline AJ68 & - & ++ & ++++ & ++ & - & ++ & ++++ & ++ & +++ & + & ++++ & +++ & ++ & + & ++++ & ++ & ++ & + \\
\hline AJ69 & - & ++ & ++++ & ++ & - & ++ & ++++ & ++ & +++ & + & ++++ & +++ & ++ & + & ++++ & ++ & ++ & + \\
\hline AJ81 & - & ++ & ++++ & ++ & - & ++ & ++++ & ++ & +++ & + & ++++ & +++ & ++ & + & ++++ & ++ & ++ & + \\
\hline AJ83 & - & ++ & +++ & ++ & - & ++ & ++++ & ++ & +++ & + & ++++ & +++ & ++ & + & ++++ & ++ & ++ & + \\
\hline AJ93 & - & ++ & +++ & ++ & - & ++ & ++++ & ++ & +++ & + & ++++ & +++ & ++ & + & ++++ & ++ & ++ & + \\
\hline
\end{tabular}




\begin{tabular}{|c|c|c|c|c|c|c|c|c|c|c|c|c|c|c|c|c|c|}
\hline \multirow{2}{*}{$\begin{array}{l}\text { Strain } \\
\text { AJ100 }\end{array}$} & \multicolumn{3}{|c|}{$\% \mathrm{NaCl}$} & \multicolumn{4}{|c|}{ pH } & \multicolumn{2}{|r|}{ UV-B (h) } & \multicolumn{4}{|c|}{ As [V] (mM) } & \multicolumn{4}{|c|}{ As [III] (mM) } \\
\hline & ++ & ++++ & ++ & - & ++ & ++++ & ++ & +++ & + & ++++ & +++ & ++ & + & ++++ & ++ & ++ & + \\
\hline AJ101 & $-\quad++$ & ++++ & ++ & - & ++ & ++++ & ++ & +++ & + & ++++ & +++ & ++ & + & ++++ & ++ & ++ & + \\
\hline AJ102 & $-\quad++$ & ++++ & ++ & - & ++ & ++++ & ++ & +++ & + & ++++ & +++ & ++ & + & ++++ & ++ & ++ & + \\
\hline AJ104 & $-\quad++$ & ++++ & ++ & - & ++ & ++++ & ++ & +++ & + & ++++ & +++ & ++ & + & ++++ & ++ & ++ & + \\
\hline AJ109 & $-\quad++$ & ++++ & ++ & - & ++ & ++++ & ++ & +++ & + & ++++ & +++ & ++ & + & ++++ & ++ & ++ & - \\
\hline AJ110 & $-\quad++$ & ++++ & ++ & - & ++ & ++++ & ++ & +++ & + & ++++ & +++ & ++ & + & ++++ & ++ & ++ & - \\
\hline AJ111 & $-\quad++$ & ++++ & ++ & - & ++ & ++++ & ++ & +++ & + & ++++ & +++ & ++ & + & ++++ & ++ & ++ & + \\
\hline AJ112 & $-\quad++$ & ++++ & ++ & - & ++ & ++++ & ++ & +++ & + & ++++ & +++ & ++ & + & ++++ & ++ & ++ & + \\
\hline AJ113 & $-\quad++$ & ++++ & ++ & - & ++ & ++++ & ++ & +++ & + & ++++ & +++ & ++ & + & ++++ & ++ & ++ & + \\
\hline AJ115 & ++ & ++++ & ++ & - & ++ & ++++ & ++ & +++ & + & ++++ & +++ & ++ & + & ++++ & ++ & ++ & + \\
\hline AJ117 & $-\quad++$ & ++++ & ++ & - & ++ & ++++ & ++ & +++ & + & ++++ & +++ & ++ & + & ++++ & ++ & ++ & + \\
\hline AJ118 & $-\quad++$ & ++++ & ++ & - & ++ & ++++ & ++ & +++ & + & ++++ & +++ & ++ & + & ++++ & ++ & ++ & + \\
\hline AJ119 & $-\quad++$ & ++++ & ++ & - & ++ & ++++ & ++ & +++ & + & ++++ & +++ & ++ & + & ++++ & ++ & ++ & + \\
\hline AJ120 & $-\quad++$ & ++++ & ++ & - & ++ & ++++ & ++ & +++ & + & ++++ & +++ & ++ & + & ++++ & ++ & ++ & + \\
\hline AJ121 & ++ & ++++ & ++ & - & ++ & ++++ & ++ & +++ & + & ++++ & +++ & ++ & + & ++++ & ++ & ++ & + \\
\hline AJ123 & $-\quad++$ & ++++ & ++ & - & ++ & ++++ & ++ & +++ & + & ++++ & +++ & ++ & + & ++++ & ++ & ++ & - \\
\hline AJ124 & $-\quad++$ & ++++ & ++ & - & ++ & ++++ & ++ & +++ & + & ++++ & +++ & ++ & + & ++++ & ++ & ++ & + \\
\hline AJ125 & $-\quad++$ & ++++ & ++ & - & ++ & ++++ & ++ & +++ & + & ++++ & +++ & ++ & + & ++++ & ++ & ++ & - \\
\hline AJ126 & $-\quad++$ & ++++ & ++ & - & ++ & ++++ & ++ & +++ & + & ++++ & +++ & ++ & + & ++++ & ++ & ++ & + \\
\hline AJ128 & ++ & ++++ & ++ & - & ++ & ++++ & ++ & +++ & + & ++++ & +++ & ++ & + & ++++ & ++ & ++ & + \\
\hline AJ130 & $-\quad++$ & ++++ & ++ & - & ++ & ++++ & ++ & +++ & + & ++++ & +++ & ++ & + & ++++ & ++ & ++ & + \\
\hline AJ131 & $-\quad++$ & ++++ & ++ & - & ++ & ++++ & ++ & +++ & + & ++++ & +++ & ++ & + & ++++ & ++ & ++ & + \\
\hline AD153 & $-\quad+$ & ++++ & ++ & - & ++ & ++++ & +++ & +++ & ++ & ++++ & +++ & ++ & + & ++++ & ++ & ++ & ++ \\
\hline AD156 & $-\quad+$ & ++++ & ++ & - & ++ & ++++ & +++ & +++ & + & ++++ & +++ & ++ & + & ++++ & ++ & ++ & ++ \\
\hline D1.1.25 & $-\quad+$ & ++++ & ++ & - & ++ & ++++ & +++ & +++ & + & ++++ & +++ & ++ & + & ++++ & ++ & ++ & ++ \\
\hline
\end{tabular}

